\title{
Rational Inconsistency and Reasoning 1
}

\author{
BRYSON BROWN University of Lethbridge
}

Key Words: Reasoning; reductio ad absurdum; inconsistency; paraconsistency; conflict; rationality. Abstract: Nicholas Rescher has argued we must tolerate inconsistency because of our cognitive limitations. He has also produced, together with $\mathrm{R}$. Brandom, a serious attempt at exploring the logic of inconsistency. Inconsistency tolerance calls for a systematic rewriting of our logical doctrines: it requires a paraconsistent logic. However, having given up all aggregation of premises, Rescher's proposal for a paraconsistent logic fails to account for the reductive reasoning Rescher appeals to in his account of inconsistency tolerance. A non-adjunctive logic developed by P.K. Schotch and R.E. Jennings provides just what Rescher's logic is lacking: It allows a tolerant attitude toward inconsistency while giving an account of reductive reasoning.

\section{I.}

Nicholas Rescher has argued persuasively for tolerating inconsistency as an inevitable, though hopefully temporary by-product of our cognitive limitations [Rescher, 1987]. He has also produced, together with $\mathrm{R}$. Brandom, a serious attempt at exploring the logic of inconsistency [Rescher and Brandom, 1980]. In this paper I examine two important questions arising from Rescher's tolerant approach to inconsistency.

Classically, every sentence is a consequence of any inconsistent set: ex falso quodlibet. So if we reason in accord with classical logic, we can't possibly countenance inconsistency. The classical recommendation, when faced with an inconsistent premise set, is to find another premise set. If we cannot or will not adopt this recommendation (and it is not a trivial problem to decide how to get from an inconsistent set of commitments to a consistent set, nor can we always do so at low cost to our other epistemic concerns), we must answer the question, just what classical principles shall we reject (at least until we have put our house in better order)? And any answer to this question leads immediately to the question, is what remains enough? Can we give a systematic account of how we should reason that both allows for tolerating inconsistency and supports the forms of reasoning we find indispensable? These questions lead us to, and beyond, The Logic of Inconsistency [Rescher and Brandom, 1980].

Both questions are staples of conservative objections to inconsistency tolerance. If ex falso quodlibet is retained, tolerating inconsistency is pure nonsense. And if we reject ex falso quodlibet, what of the rest of our reasoning? Inconsistency tolerance calls for a systematic rewriting of our logical doctrines. However, this re-writing must remain faithful to our day-to-day practice of argument. This double challenge, to change and preserve, calls for both philosophical and technical agility.

\section{In praise of The Logic of Inconsistency}

Rescher and Brandom [1980] propose a straightforward answer to the first question. They suggest we give up conjunction introduction, the rule that tells us that any two sentences imply their conjunction. ${ }^{2}$ The result is that inconsistent sets which do not include a contradiction no longer imply everything. In fact, the conse- 
quences of any set now become simply the individual consequences of the separate sentences it contains. Conjunction introduction is the principle of classical logic which allows us to "aggregate" the force of separate premises. Without it (so long as we have no other principles of aggregation) each premise contributes only its own isolated consequences.

Having rejected conjunction introduction, Rescher and Brandom are able to distinguish two facts which classical logic runs together:

1. $\{A, B, C, D \ldots\}$ implies $P$.

2. (A \& B \& C \& D ...) implies $P$.

In classical logic conjunction introduction gets us from 1 to 2 by conjoining all of A,B,C,D together. But without it, 1 simply means that either $A$ implies $P$, or $B$ implies $P$, or $C$ implies $P$, or.... Thus 2 can hold without 1 holding.

If we accept Rescher and Brandom's proposal, the implications of a set become the implications of its individual members. When $A$ and $B$ are contrary sentences, the conjunction ( $\mathrm{A} \& \mathrm{~B} \& \mathrm{C} \& \mathrm{D}$...) classically implies every sentence: Its consequences are trivial. But so long as none of $A, B, C, D$, etc. is a contradiction, the set $\{A, B, C, D, \ldots\}$ will have non-trivial consequences, i.e. some sentences will be consequences of the set and some sentences will not.

So we no longer have ex falso quodlibet-instead we have ex falso quodlibet "lite". ${ }^{4}$ The logic that results is nearly classical, and very simply applied. We dispense with all classical implications that depend on more than one premise while retaining all the classical implications with only one premise. 5

Another important virtue of this approach is that it captures normal contextual constraints on how we reason. We don't go around conjoining things willynilly - to do so would be largely a waste of time, and in some circumstances worse than that. In fact we are quite selective about what cognitive commitments we bring to bear in any given circumstance. While measuring the dimensions of a room, I may apply some knowledge of Euclidean geometry, but I won't make use of what I know about the chemical composition of water. And cognitive commitments that are never brought to bear together need never be conjoined-they have separate existences, quite independent of each other. This has practical consequences for logic, since it means that commitments that are never brought to bear together need not be logically consistent with each other.

I reason in every respect as though I believe in Euclidean geometry when I am measuring the room-I have no other way of doing the job. Yet if I am asked to discuss the path of a light ray in the neighbourhood of a large mass, I will appeal to general relativity - a very different sort of geometry. The two are inconsistent with each other, but the fact that I use them in entirely separate contexts makes this fact harmless. Rescher and Brandom's rejection of conjunction introduction allows us to treat what.is obviously practically coherent as also logically coherent.

In [1987] Rescher raises a problem of practical reasoning in the face of inconsistency that responds to similar treatment. The puzzle is about whether we should always act on our beliefs. If we should then it seems we should bet on their truth if we are to receive some small gain on winning even if the price of losing is our lives and and more, even when we could choose instead not to bet, or to take the other side of the bet. Rescher responds to the problem by distinguishing two different sorts of commitment: $\mathrm{C}$-commitment, the sort of commitment we make to claims we are prepared to stake anything and everything on, and a more diffident sort of commitment, which we make to claims we regard simply as likely parts of the true world story. The latter we may believe without being committed to act on them in any and every circumstance. In particular, 
when the stakes are high enough, we may conservatively "pull in our horns" and refuse to act on these commitments for fear of the consequences if they turn out to be false after all.

One way to express this distinction is to think about two different sorts of practical context. In one we are concerned to express our views of the world, to articulate the best "world story" we can construct. In the other we are concerned to achieve more concrete ends by placing bets attached more or less arbitrarily to the truth or falsity of various claims. Depending on the stakes offered, commitments that we gladly bring to bear in the first might be utterly absurd in the second, and vice-versa. Obviously we won't normally be willing to conjoin commitments appropriate in the first sort of context with commitments appropriate in the second.

\section{A fly in the ointment}

There is a problem, however. Rescher and Brandom's approach makes nothing of relations between sentences in a set of premises. This makes it very difficult to understand what I call "reductio" reasoning. Reductio reasoning works by testing a sentence against a background of accepted sentences-when we can derive a contradiction we either do not admit the sentence to the premise set, or we remove some other sentence to prevent the premise set from becoming inconsistent. We can understand this classically - after all, adding such a sentence will trivialize the premise set's consequences. We can even give a simple definition of absurdity with respect to a set of sentences: A sentence is absurd with respect to a set of sentences if and only if the set by itself has a non-trivial consequence set, but with the sentence added has a trivial consequence set.

However, in Rescher and Brandom's system only contradictions are absurd in this sense, and they are absurd with respect to every set. Adding a sentence to a set merely adds it, together with its logical consequences, to the set's consequences. And this goes for any set: Adding a noncontradictory sentence cannot render a set trivial, so no sentence whose individual consequences aren't trivial is absurd with respect to any set. Rescher and Brandom's approach is utterly insensitive to the logical relations between members of the premise set. But reductio reasoning, whatever detailed account of it is right, clearly must turn on relations between the premise we add and the rest of the premise set.

Retaining some account of this sort of relative reductio is necessary if we want to understand why we go about rejecting acceptance-candidates, or rejecting previously accepted claims, when an acceptance candidate conflicts with the previously accepted claims. But we cannot retain relative reductio while tolerating inconsistency in the way Rescher proposes. Relative reductios turn on logical or more broadly inferential relations between a candidate for acceptance and sentences already accepted, and those relations are lost to Rescher and Brandom's logic.

Relative reductio also plays a role in the reductive reasoning that Rescher makes central to his case for tolerating inconsistency [1987, 307-313]. This sort of reasoning doesn't involve comparing new candidates for acceptance with other already accepted claims. Instead, Rescher focuses on finding ways to cut down our initially inconsistent tentative commitments. But relations between accepted claims, and in particular incompatibilities between them are central in such reasoning. So by giving up all aggregation of premises, Rescher's concrete proposal for a paraconsistent logic abandons any explanation of the reductive reasoning central to Rescher's case for inconsistency tolerance.

Happily, a proposal by P.K. Schotch and R.E. Jennings ${ }^{6}$ comes to the rescue. They have developed a non-adjunctive logic closely related to Rescher and Bran- 
dom's, which does not give up all aggregation of premises. Instead, Schotch and Jennings give up just enough aggregation to avoid trivialization. The result is that when we add a sentence which requires us to give up even more aggregation, the set trivializes. Thus the added claim is absurd with respect to the set, in a sense that is a natural generalization of the classical account of relative absurdity above. I'll describe Schotch and Jennings" system briefly to explain how this works, without dwelling on the formal details any more than necessary.

Schotch and Jennings' logic turns on dividing the premises up into classically consistent compartments or cells. If we can do this with just one cell, then the premise set is classically consistent and we can use the classical aggregation rule, conjunction introduction. If we need two cells, then we replace conjunction introduction with $2 / 3$ introduction: This rule tells us to infer from any three premises (or any three consequences of premises) the disjunction of their pairwise conjunctions: Applied to the triple $A, B, C, 2 / 3$ introduction gives us $(A \& B) \vee(A \& C) \vee(B \& C)$. After all, if we are distributing $A, B$, and $C$ among just two cells, one of these pairs must end up together in the same cell. If we need 3 cells, we use $2 / 4$ introduction, inferring the disjunction of the pairwise conjunctions amongst all groups of $4 .{ }^{7}$ In general, if we need $n$ cells to divide the premises into consistent groups, Schotch and Jennings' logic allows us to use $2 / n+1$ to aggregate premises. The number of cells required to get a consistent division of a premise set is called the premise set's level of incoherence.

We can now give a definition for relative absurdity:

$P$ is absurd relative to $\{A, B, C, \ldots\}$ iff $\{A, B, C \ldots\}$ has level $l$ and $\{A, B, C, \ldots, P\}$ has level $l$ and $l>l$.

In plain English, a sentence is absurd with respect to some premise set if and only if adding it to the set increases the set's level. This definition makes use of the sentence's logical relations to other members of the set-adding a sentence to a premise set in Schotch and Jennings' system adds consequences that depend on aggregations of the new premise with other premises. Sentences that are not contradictions, and that are perfectly compatible with some premise sets, will still increase the level of other premise sets.

In practical terms, adding such a sentence to our commitment set requires us to divide up contexts of application for our commitments more finely than we had. The cost of doing this, and the difficulty of doing it in a way that captures any natural division of practical contexts, makes adding such level-increasing sentences very costly. Schotch and Jennings' logic provides a plausible account of both our ability to tolerate inconsistency and our use of relative reductio arguments to constrain the addition of new sentences to our commitments.

Schotch and Jennings' logic also accounts for the reductive reasoning that Rescher describes. This sort of reasoning takes place when we recognise our initial commitments are less coherent than we initially thought they were, and we respond by examining ways of reducing our commitments, trying to find the least costly way of doing so.

The only reasoning of this sort that classical logic can capture occurs when our initial commitments are suddenly recognised to be inconsistent. At that point classical reasoning must be suspended until we have restored consistency. But as the history of old quantum theory shows, reasoning continues even when our commitments are inconsistent. This much Rescher and Brandom can account for. But as the history of old quantum theory also shows, when we decide to tolerate inconsistency, we do so at a specific level, in a way guided concretely by policies for separating the contexts of 
application of our inconsistent premises. And the later collapse of old quantum theory was brought on, at least in part, by the discovery that the inconsistency of old quantum theory was worse than we thought: Pauli showed that the commitments of old quantum theory together with the accepted constraints on contexts of application allowed us to prove an absurdity when applied to a hydrogen atom in crossed electrical and magnetic fields. ${ }^{8} \mathrm{~A}$ finer division of contexts of application was needed to save old quantum theory. Rescher and Brandom's logic makes this historical case hard to understand: why should we worry about how finely we have to divide contexts of application if all aggregation of premises is out anyway? But Schotch and Jennings' approach is more helpful, Scientists had treated old quantum theory as a level 2 theory; Pauli showed the theory's level was at least 3 :
There is a clear logical problem in the theory's commitments regarding the states of hydrogen atoms in crossed fields.

\section{Conclusions}

Schotch and Jennings' non-adjunctive system provides just what Rescher and Brandom's logic is lacking. It supports the tolerant attitude toward inconsistency that Rescher advocates, while preserving relative-reductio reasoning. The history of old quantum theory underwrites the importance of the concept of levels of incoherence, which allows us to explain the importance of Pauli's demonstration that the adiabatic principle conflicts with other commitments of the quantum theory. Schotch and Jennings' approach makes the difficulty clear, while allowing us to tolerate inconsistency as Rescher argues we should.

\section{Notes}

1 I want to thank the SSHRC of Canada (grant 410-89-0633) for supporting the research that led to this paper.

2 Rescher and Brandom's approach is semantically driven-they construct models for inconsistent sets of sentences by "superposing" pairs of classical possible worlds. A superposed world satisfies a sentence if either of the worlds it is built from does. Thus if one of the worlds satisfied " $A$ " and another satisfied "not $A^{\prime}$, the superposed world would satisfy both

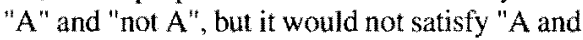
not $A^{\prime \prime}$, since neither of the original worlds did.

See Rescher and Brandom [1980], 16 ff. Rescher and Brandom insist that they are offering a non-standard semantics, and not a non-standard logic. This follows from their claim that our actual world must be a consistent and complete world. As a result, the actual truth of $P \& Q$ does follow from the actual truth of $P$ and of $Q$. Conjunction introduction is therefore sound for the actual world. But the sentences we accept may be inconsistent, and reasoning with them is best conducted on the assumption that they are all true in some nonstandard world and then applying Rescher and Brandom's approach to determine what else must be true in such a world.

This approach, in which contradictions remain beyond the pale, should be contrasted with the much more radical approach to paraconsistency of the Australian and Brazilian schools, both of which countenance true contradictions and reject any form of ex falso quodlibet. See G. Priest, R. Routley and J. Norman, eds. [1989] for an overview of various paraconsistent logics.

5 It's worth pointing out here that this is the response $\mathrm{H}$. Kyburg has advocated to the socalled lottery paradox ever since he first discovered it: Kyburg (for example, in [1983]) rejects conjunction introduction because it results (in general) in conclusions that are less probable than the premises. Conclusions that are extremely improbable can be reached if we begin from enough independent, highly probable premises. 
6 See Schotch and Jennings [1980] and [1989] for more details.

7 See Schotch and Jennings [1989] and Apostoli and Brown, forthcoming, for proofs of compactness and completeness, and further discussion of related issues.
8 See Mehra and Rechenberg [1982] 508-9 for a discussion of this result and its impact on old quantum theory.

\section{Bibliography}

Apostoli and Brown, "Compactness and Completeness for Generalized Modal Logics", forthcoming.

Kyburg, $\mathrm{H}$., Epistemology and Inference, (University of Minnesota Press, Minneapolis, 1983).

Mehra, J., and Rechenberg, H., The Historical Development of Quantum Theory, Volume 1, parts $I$ and 2 (New York, Springer-Verlag, 1982)

Priest, G., Routley, R., and Norman, J., Paraconsistent Logic: Essays on the Inconsistent, (Philosophia Verlag, Hamden, Munchen, Wien, 1989).

Priest, G., Beyond Consistency, (Philosophia Verlag, Hamden, Munchen, Wien, 1988)
Rescher, N. and Brandom, R., The Logic of Inconsistency, (Basil Blackwell, Oxford, 1980).

Rescher, N., "How Serious a Fallacy is Inconsistency?", Argumentation, 1,3, (1987) 303-16.

Schotch, P.K., and Jennings, R.E., "Inference and Necessity", Journal of Philosophical Logic, 9 (1980) $327-40$.

Schotch, P.K., and Jennings, R.E., "On Detonating", 302-27 in Priest, Routley and Norman, eds. [1989]

BRYSON BROWN

DEPARTMENT OF PHILOSOPHY

UNIVERSITY OF LETHBRIDGE

LETHBRIDGE, ALBERTA TIK $3 M 4$

\section{Thinking: The Journal of Philosophy for Children}

\section{ARTICIES IN RECENT ISSUES}

EKKEHARD MARTENS, Philosophy for Children and Continental Philosophy

DAVD KENNEDY, Fools, Young Children and Philosophy

CHRISTINA SLADE, Logic in the Classroom

ALIAN COLLINS et. al., Cognitive Apprenticeship

PATRICK J.M. COSTELlO, Akrasia and Animal Rights
WOLFE MAYS, Thinking Skills Programs: An Analysis

STAN ANIH, Nigerian College Adopts

"Community of Inquiry" Approach JOSEP CULLEL, Philosophy for Children in Catalonia

JOHN WILSON, Philosophy for Children: A Note of Warning

RICHARD J. BERNSTEIN, Does Philosophy Matter?

plus, in every issue, a book review by GARETH B. MATTHEWS, a report on education for thinking in North America by BARRY BEYER, and a report on education for thinking in Europe by JOHN NISBET.

Thinking is published four times a year. Individual subscriptions $\$ 25.00$, institutional subscriptions $\$ 40.00$. Foreign subscriptions add $\$ 10.00$ for postage; U.S. subscriptions add $\$ 5.00$ for postage.

Correspondence should be addressed to: Thinking, Institute for the Advancement of Philosophy for Children, Montclair State College, Upper Montclair, New Jersey 07043. 